\title{
A Decision Procedure for Regular Membership and Length Constraints over Unbounded Strings ${ }^{\star}$
}

\author{
Tianyi Liang ${ }^{1}$, Nestan Tsiskaridze ${ }^{1}$, Andrew Reynolds ${ }^{2}$, \\ Cesare Tinelli ${ }^{1}$, and Clark Barrett ${ }^{3}$ \\ 1 Department of Computer Science, The University of Iowa \\ 2 École Polytechnique Fédérale de Lausanne \\ 3 Department of Computer Science, New York University
}

\begin{abstract}
We prove that the quantifier-free fragment of the theory of character strings with regular language membership constraints and linear integer constraints over string lengths is decidable. We do that by describing a sound, complete and terminating tableaux calculus for that fragment which uses as oracles a decision procedure for linear integer arithmetic and a number of computable functions over regular expressions. A distinguishing feature of this calculus is that it provides a completely algebraic method for solving membership constraints which can be easily integrated into multi-theory SMT solvers. Another is that it can be used to generate symbolic solutions for such constraints, that is, solved forms that provide simple and compact representations of entire sets of complete solutions. The calculus is part of a larger one providing the theoretical foundations of a high performance theory solver for string constraints implemented in the SMT solver CVC4.
\end{abstract}

\section{Introduction}

The study of word algebra and regular expressions has a long history in mathematics and computer science. There has been much renewed interest lately for these topics within the software verification and computer security communities because of the increasing importance of reasoning about character strings and regular expressions when proving safety properties or trying to detect security violations in programs that process string values.

To support these applications, several systems have been developed recently that check the satisfiability of constraints over a rich set of string operations including string equalities and inequalities, string length, regular language membership, and additional functions over strings besides string concatenation [34, 1, 19, 30. A lot of this work focuses on generally (refutation) incomplete methods to detect the unsatisfiability of these constraints, a practical approach for making progress in program analysis applications. A major difficulty in providing complete methods is that any reasonably comprehensive theory of character strings

* This work was partially funded by NSF grants \#1228765 and \#1228768. 
is undecidable [7, 23, 27]. However, several more restricted, but still quite useful, theories of strings do have a decidable satisfiability problem. These include any theories of fixed-length strings, which are trivially decidable because their domains are finite, but also some fragments over unbounded strings (e.g., word equations [22, 25]). Recent research has focused on identifying decidable fragments suitable for program analysis and, more crucially, on developing efficient solvers for them.

In previous work, we described a comprehensive approach, based on algebraic techniques and described abstractly as a calculus, to reason efficiently about quantifier-free formulas in a rich theory of unbounded strings with length and regular language membership [19]. And based on that approach, we constructed an efficient string solver, fully integrated into the multi-theory SMT solver CVC4. The calculus developed in that work is both refutation and solution sound but refutation incomplete.

Contribution and significance We have developed an improved version of the calculus presented in [19] that is also complete and terminating over a restriction of the general language to membership and length constraints. In this paper, we present a simplified version of that calculus which can be used to prove that the fragment in question is decidable. Strictly speaking, this decidability result is not new, as it is implicitly implied by some recent results from Abdulla et al. 1], although that work does not mention the result. We provide a full proof based on the calculus presented here. This contribution is significant not only because of the importance of the fragment but also for the following reasons. First, contrary to previous approaches for solving membership constraints which rely on reductions to finite state automata problems, our approach is completely algebraic and works directly with regular expressions. This facilitates the creation of efficient incremental solvers which can be more easily incorporated into modern SMT solvers since they do not rely on eager conversion to automata problems. Second, our completeness argument shows how to produce symbolic solutions for satisfiable problems with regular membership constraints, that is, intensional representations of (possibly infinite) sets of concrete solutions. This is useful for security analysis applications like filter generation and automatic exploit generation (AEG), where any assignment satisfying the constraints generated from a program is a security exploit. A symbolic solution enables AEG applications, for example, to generate fewer, more general exploits, thus also reducing the number of exploits that would need to be examined by a user.

Although our eventual goal is overall efficiency in practice, the calculus presented here focuses (for simplicity) on proving the decidability result. As a consequence, it uses a few auxiliary functions that apply generally inefficient eager (but algebraic) conversions from and to regular expressions. We plan to present in future work a version of the calculus that lifts these conversions to a set of additional derivation rules, making them amenable to lazy and selective application based on search heuristics. 


\subsection{Related work}

There have been a number of different approaches for solving string constraints with regular expressions. The earliest and perhaps most established approach is based on reductions to automata decision problems. One of these was implemented in the system DPRLE, used to check programs against SQL injection vulnerabilities [13. The approach followed in that system has the strong limitation of imposing an upper bound on the length of string variables, a hard to overcome drawback shared by various later works. This approach was later improved by the same author with a method for generating automata lazily from the input problem which does not requiring any priori length bounds [14. At the same time, a comprehensive set of algorithms and data structures for performing fast automata operations was developed to support constraint solving over strings, for instance in [12.

Current automata-based approaches to reason about regular expressions can be divided in two classes depending on whether their transitions processing a single character a time (e.g., [9, 33]) or a set of them (e.g., 31, 32, 14]). Most of the tools based on these approaches offer very limited support to reason about constraints mixing strings and other data types. Also, automata refinement may constitute a performance bottleneck, even though it is very useful in solving membership constraints. Further discussion can be found in [10, 18. Other approaches for solving regular expression constraints are based on reductions to other theories, such as bit-vectors [15] or linear integer arithmetic constraints [29], 7], and using constraint solvers for those theories.

Three notable systems that solve regular membership constraints are REX [32, 31, Mona [1] and the Java String Analyzer (JSA) 8]. REX too is based on automata. In contrast to the work described in [14] where each transitions covers an integer interval, Rex encodes strings as symbolic finite automata (SFA) first. Each SFA transition uses a logical predicate over linear arithmetic to represent a set of character-level candidates. This allows REx to encode transitions as SMT constraints which it then sends to an SMT solver for a model. This approach provides an efficient encoding for solving membership constraints, however, it currently does not support mixed constraints over additional theories.

MonA is a solver for monadic second-order logic with built-in support for string constraints. Although MoNA is an automata-based, it uses Multi Terminal BDDs to represent automata. This kind of implementation requires sophisticated engineering techniques (see [16]) which make it difficult to build in additional theories to support solving of combined constraints. PISA [28] is another string solver based monadic second-order logic. However, the language of PISA is rather restrictive, e.g., no binary operations between two variables are allowed.

JSA is geared specifically to Java string constraints. It first translates them to a flow graph, and then analyzes the graph by converting it to a context-free language. This language is approximated with the Mohri-Nederhof algorithm to a regular one and encoded as a multi-level automaton. Compared to our work, JSA focuses exclusively on Java string analysis, approximation, and automaton 
conversion, while our approach does not depend on any particular language, and solves string constraints natively with no approximations.

It is well-known that regular languages are closed under common operations (e.g., concatenation, union, intersection, complementation); however, the complexity of performing most of these operations is high as a consequence of the high complexity of the corresponding membership problem. For example, membership in the intersection of two regular languages is PSPACE-complete [17. Thus, in practice many procedure implementing regular language operations are approximate (e.g., [6, 26]). In contrast, the calculus we present here does not approximate.

Our calculus decides a fragment that combines regular membership constraints with string length constraints. To the best of our knowledge, there are no explicit claims about the decidability of this fragment. The work in [1] implies that the fragment is indeed decidable, although the paper contains no proof, or mention, of this. The method described in that paper replaces all characters in regular expressions with a single arbitrary character, and reduces the expression to their Parikh images 24, generating a set of semi-linear integer constraints which can then be checked for satisfiability using any linear arithmetic solver. Since our approach does not use rely on approximations it can build a model directly when the constraints are satisfiable. This part of work our has some similarities with the Parikh image described in 4, although we developed it independently.

\subsection{Formal preliminaries}

We work in the context of many-sorted first-order logic with equality $(\approx)$. We assume the reader is familiar with the notions of many-sorted signature, term, literal, formula, free variable, interpretation, and satisfiability of a formula in an interpretation (see, e.g., [5] for more details). A theory is a pair $T=(\Sigma, \mathbf{I})$ where $\Sigma$ is a signature and $\mathbf{I}$ is a class of $\Sigma$-interpretations, the models of $T$, that is closed under variable reassignment. If $\mathcal{I}$ is an interpretation and $t$ is a term, we denote by $t^{\mathcal{I}}$ the value of $t$ in $\mathcal{I}$. A $\Sigma$-formula $\varphi$ is $T$-satisfiable (resp., $T$-unsatisfiable) if it is satisfied by some (resp., no) interpretation in I. A set $\Gamma$ of formulas entails in $T$ a $\Sigma$-formula $\varphi$, written $\Gamma=_{T} \varphi$, if every interpretation in $\mathbf{I}$ that satisfies all formulas in $\Gamma$ satisfies $\varphi$ as well. The set $\Gamma$ is satisfiable in $T$ if $\Gamma \nvdash_{T} \perp$ where $\perp$ is the universally false atom. If $e$ is a term or a formula, we denote by $\mathcal{V}(e)$ the set of $e$ 's free variables, extending the notation to sets of terms or formulas as expected. Two $\Sigma$-formulas $\varphi$ and $\psi$ are $T$-equisatisfiable if for every model $\mathcal{I}$ of $T$ that satisfies one, there is a model of $T$ that satisfies the other and differs from $\mathcal{I}$ at most over the free variables not shared by $\varphi$ and $\psi$.

\section{A theory of strings and regular language membership}

We consider a theory $T_{\mathrm{LR}}$ of strings with length and regular language membership constraints over a signature $\Sigma_{\mathrm{LR}}$ with three sorts, Str, Int, and Lan, and an infinite 


$$
\begin{aligned}
& \epsilon: \operatorname{Str} \quad-_{-}{ }_{-} \text {Str } \times \operatorname{Str} \rightarrow \operatorname{Str} \quad c: \operatorname{Str} \text { for all } c \in \mathcal{A} \quad\left|{ }_{-}\right|: \operatorname{Str} \rightarrow \operatorname{Int}
\end{aligned}
$$

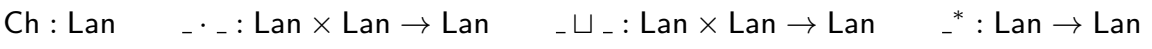

$$
\begin{aligned}
& \varnothing: \text { Lan } \quad \text { - in } \_: \operatorname{Str} \times \operatorname{Lan} \quad-\Pi_{-}: \operatorname{Lan} \times \operatorname{Lan} \rightarrow \operatorname{Lan} \quad\left\ulcorner_{-}\right\urcorner: \operatorname{Str} \rightarrow \operatorname{Lan}
\end{aligned}
$$

Fig. 1. Basic set of string and regular expression function and predicate symbols.

$$
()^{(-)}: \text {Lan } \times \operatorname{Int} \rightarrow \text { Lan } \quad \text { sh }: \text { Lan } \times \text { Lan } \times \text { Lan } \rightarrow \text { Lan }
$$

Fig. 2. Additional regular expression function symbols.

set of variables for each of these sorts. This theory is essentially the theory of a single many-sorted structure and its models differ only on how the variables are interpreted. All models of $T_{\mathrm{LR}}$ interpret Int as the set of integer numbers, Str as the language $\mathcal{W}$ of all words over some fixed finite alphabet $\mathcal{A}$ of characters, and Lan as the power set of $\mathcal{W}$. The signature includes: the usual symbols of linear integer arithmetic, interpreted as expected; all the elements of $\mathcal{W}$ as constant symbols, or string constants, interpreted as themselves; and all the function symbols given in Figure 1 with their rank. In that figure, the two - symbols denote word concatenation and language concatenation, respectively; |-| denotes word length; and \ulcorner\urcorner denotes the singleton set constructor, mapping each word $w \in \mathcal{W}$ to the language $\{w\}$; the symbols $\epsilon, \mathrm{Ch}, \varnothing$, and in respectively denote the empty word, the language of one-character words, the empty language, and the language membership predicate; the symbols $\sqcup, \sqcap$, and (_)* respectively denote language union, intersection and Kleene closure.

We call a string term any term of sort Str or of the form $|s|$; an arithmetic term any term of sort Int all of whose occurrences of $\left.\right|_{-} \mid$are applied to a variable; and a regular expression any variable-free term of sort Lan. A string term is atomic if it is a variable or a string constant. An arithmetic constraint is a (dis)equality $(\neg) u \approx v$ or an inequality $u \geq v$ where $u$ and $v$ are arithmetic terms. A membership constraint is a literal of the form $(\neg)(s \in r)$ where $s$ is a string term and $r$ is a regular expression. A $T_{\mathrm{LR}}$-constraint is an arithmetic or a membership constraint. Note that we do not consider here equalities between terms of sort Str. Also note that if $x$ is a string variable, $|x|$ is both a string and an arithmetic term. By the definition of $T_{\mathrm{LR}}$, a regular expression $r$ is interpreted as the same language in every model of $T_{\mathrm{LR}}$. We call that the language generated by $r$ and denote it by $\mathcal{L}(r)$.

Expanding the language The calculus we present later is able to compute a solved form for a satisfiable input set of $T_{\mathrm{LR}}$-constraints with string variables $x_{1}, \ldots, x_{n}$. This solved form consists of a set $\left\{x_{i} \text { in } q_{i}\right\}_{i=1, \ldots, n}$ of membership constraints where, for all $i, q_{i}$ is a solved-form term, a term of sort Lan over integer variables and a signature that includes string constants, the symbols $\mathrm{Ch}$, $\cdot$ and \ulcorner\urcorner$_{-}$from Figure 1, and the two function symbols from Figure 2. Note that the latter two symbols are not in the (input) language of $T_{\mathrm{LR}}$-constraints; they are used only in solved forms. We expand the models of $T_{\mathrm{LR}}$ to these two symbols so that the following holds. 


$$
\begin{aligned}
& \left(s_{1} \cdot s_{2}\right) \cdot s_{3} \rightarrow s_{1} \cdot\left(s_{2} \cdot s_{3}\right) \\
& s \cdot \epsilon \rightarrow s \quad \epsilon \cdot s \rightarrow s \\
& \left|s_{1} \cdot s_{2}\right| \rightarrow\left|s_{1}\right|+\left|s_{2}\right| \quad|c| \rightarrow 1 \quad|\epsilon| \rightarrow 0 \\
& r_{1} \cdot\left(r_{2} \sqcup r_{3}\right) \rightarrow\left(r_{1} \cdot r_{2}\right) \sqcup\left(r_{1} \cdot r_{3}\right) \quad\ulcorner\epsilon\urcorner \cdot r \rightarrow r \quad \varnothing \cdot r \rightarrow \varnothing \\
& \left(r_{1} \sqcup r_{2}\right) \cdot r_{3} \rightarrow\left(r_{1} \cdot r_{3}\right) \sqcup\left(r_{2} \cdot r_{3}\right) \quad r \cdot\ulcorner\epsilon\urcorner \rightarrow r \quad r \cdot \varnothing \rightarrow \varnothing \\
& \left\ulcorner s_{1}\right\urcorner \cdot\left\ulcorner s_{2}\right\urcorner \rightarrow\left\ulcorner s_{1} \cdot s_{2}\right\urcorner \quad r^{* *} \rightarrow r^{*} \quad\ulcorner\epsilon\urcorner^{*} \rightarrow\ulcorner\epsilon\urcorner \\
& r \sqcup r \rightarrow r \quad(r \sqcup\ulcorner\epsilon\urcorner)^{*} \rightarrow r^{*} \quad \varnothing^{*} \rightarrow\ulcorner\epsilon\urcorner \\
& r_{1} \sqcap r_{2} \rightarrow \pi\left(r_{1}, r_{2}\right) \quad \varnothing \sqcup r \rightarrow r \quad \varnothing \sqcap r \rightarrow \varnothing
\end{aligned}
$$

Fig. 3. Term normalization rules, defined modulo commutativity of $\sqcup$ and $\sqcap$; $\pi\left(r_{1}, r_{2}\right)$ is the regular expression computed by the function $\pi$ defined in Figure 8.

- For all integers $n$ and regular expressions $r, \mathcal{L}\left(r^{n}\right)=\{\epsilon\}$ if $n \leq 0$ and $\mathcal{L}\left(r^{n}\right)=\mathcal{L}\left(r \cdot r^{n-1}\right)$ otherwise.

- For all regular expressions $r, r^{\prime}, q, \mathcal{L}\left(\operatorname{sh}\left(r, r^{\prime}, q\right)\right)=\left\{w_{1} w_{1}^{\prime} \cdots w_{n} w_{n}^{\prime} \in \mathcal{L}(q) \mid\right.$ $\left.n>0, w_{1} \cdots w_{n} \in \mathcal{L}(r), w_{1}^{\prime} \cdots w_{n}^{\prime} \in \mathcal{L}\left(r^{\prime}\right)\right\} !^{4}$

Intuitively, the strings generated by $\operatorname{sh}\left(r, r^{\prime}, q\right)$ can be obtained by shuffling together a word $w$ generated by $r$ and a word $w^{\prime}$ generated by $r^{\prime}$, as long as the resulting word is in the language generated by $q$. Shuffling is achieved by breaking $w$ and $w^{\prime}$ arbitrarily into $n$ segments and merging the two lists of segments together.

Notational conventions We use $c, d$ to denote character constants, that is, string constants of length one; $l$ for arbitrary string constants; $x$ for string variables; $s, t$ for string terms; $z$ for integer variables; $u, v$ for arithmetic terms; and $q, r$ for regular expressions. We will omit applications of the $\ulcorner$ $\urcorner$ operator, treating (variable-free) terms of sort Str as the corresponding regular expression. When convenient, we will treat a multi-character constant $l$ as the term $c \cdot l^{\prime}$ where $c$ is the first character of $l$ and $l^{\prime}$ is the rest of $l$. We will write $\models_{\mathrm{LR}}$ instead of $\models_{T_{\mathrm{LR}}}$.

\section{A calculus for constraint satisfiability in $T_{\mathrm{LR}}$}

We are interested in checking the satisfiability in $T_{\mathrm{LR}}$ of finite sets of $T_{\mathrm{LR}^{-}}$ constraints as defined in Section 2. In this section, we describe a tableaux-style calculus that can be used to construct a decision procedure for this problem.

Configurations The calculus applies to a finite set of $T_{\mathrm{LR}}$-constraints with the goal of determining their $T_{\mathrm{LR}}$-satisfiability. It consists of derivation rules that operate over configurations. A configuration is either the distinguished configuration unsat or a tuple of the form $\langle A, R, V\rangle$, where: $A$ is a set of arithmetic constraints and implications of the form $z_{1} \approx 0 \Rightarrow z_{2} \approx 0 ; R$ is a set of positive membership constraints; and $V$ is a set of membership constraints in solved form.

4 Any of the words $w_{1}, \ldots, w_{n}, w_{1}^{\prime}, \ldots, w_{n}^{\prime}$ in the definition of sh could be empty. We use juxtaposition to denote word concatenation at the semantic level. 


$$
\begin{aligned}
& \text { A-Conflict } \frac{\mathrm{A} \models \text { LIA } \perp}{\text { unsat }} \quad \text { EmptyS } \frac{\epsilon \text { in } r \in \mathrm{R} \operatorname{not} \varepsilon(r)}{\text { unsat }} \quad \text { EmptyR } \frac{s \text { in } \varnothing \in \mathrm{R}}{\text { unsat }} \\
& \begin{array}{l}
\mathrm{R}=R, x \text { in } l \\
\text { Assign-1 } \frac{\mathrm{R}:=(R\{x \mapsto l\}) \downarrow \quad \mathrm{V}:=\mathrm{V}, x \text { in } l}{\mathrm{~A}:=\mathrm{A},|x| \approx|l| \downarrow \quad}
\end{array} \\
& \text { Assign-2 } \frac{\mathrm{R}=R, x \text { in } r \quad x \notin \mathcal{V}(R) \quad \operatorname{top}(r) \notin\{\sqcup, \varnothing\} \quad \gamma(r)=(q, u, A)}{\mathrm{A}:=\mathrm{A},|x| \approx u \downarrow, A \downarrow \quad \mathrm{R}:=R \quad \mathrm{~V}:=\mathrm{V}, x \text { in } q} \\
& \text { Consume- } 1 \frac{\mathrm{R}=R, c \text { in } r}{\mathrm{R}:=R, \epsilon \text { in }\left(\partial_{c} r\right) \downarrow} \quad \text { Consume }-2 \frac{\mathrm{R}=R, c \cdot s \text { in } r}{\mathrm{R}:=R, s \text { in }\left(\partial_{c} r\right) \downarrow} \\
& \text { Split } \frac{\mathrm{R}:=R, x \cdot s \text { in } r}{\|_{\left(r_{1}, r_{2}\right) \in \beta(r)} \mathrm{R}:=R, x \text { in } r_{1 \downarrow}, s \text { in } r_{2} \downarrow} \\
& \text { Inter } \frac{\mathrm{R}:=R, s \text { in } r_{1}, s \text { in } r_{2}}{\mathrm{R}:=R, s \text { in }\left(r_{1} \sqcap r_{2}\right) \downarrow} \quad \text { Union } \frac{\mathrm{R}:=R, s \text { in } r_{1} \sqcup r_{2}}{\mathrm{R}:=R, s \text { in } r_{1} \quad \| \quad \mathrm{R}:=R, s \text { in } r_{2}}
\end{aligned}
$$

Fig. 4. Derivation Rules. $R\{x \mapsto l\}$ is the result of applying the substitution $\{x \mapsto l\}$ to every term in $R$; $\operatorname{top}(r)$ is the top symbol of term $r$.

Informally, the sets $A$ and $R$ initially store a $T_{\mathrm{LR}}$-equisatisfiable variant of the input set and progressively receive additional constraints derived by the calculus; $V$, which is initially empty, represents the solution computed so far (each string variable in $V$ is associated with a set of possible values using solved-form terms).

By standard transformations, one can convert any finite set of $T_{\mathrm{LR}}$-constraints into a $T_{\mathrm{LR}}$-equisatisfiable set $A \cup R$ where $R$ is a set of positive membership constraint 5 and $A$ is a set of arithmetic constraints that includes a constraint of the form $|x| \geq 0$ for every string variable $x \in \mathcal{V}(A)$ and contains no string variables that do not occur in $R$. We assume that all terms in such configurations are irreducible by the rewrite system in Figure 3 which can be shown to be equivalence-preserving and terminating over $\Sigma_{\mathrm{LR}}$-terms ${ }^{6}$ The rewrite system uses the auxiliary function $\pi$, closely based on one by $\mathrm{Lu} \mathrm{[21],} \mathrm{which} \mathrm{maps} \mathrm{two}$ regular expressions $r_{1}$ and $r_{2}$ to a regular expression that generates the same language as $r_{1} \sqcap r_{2}$ (i.e., $\left.\mathcal{L}\left(\pi\left(r_{1}, r_{2}\right)\right)=\mathcal{L}\left(r_{1} \sqcap r_{2}\right)\right)$ but contains no occurrences of $\sqcap$. If $t$ is a $\Sigma_{\mathrm{LR}}$-term, we denote by $t \downarrow$ any normal form of $t$ with respect to the rewrite system in Figure 3 , and extend this notation to sets of $\Sigma_{\mathrm{LR}}$-terms as expected. We call a term $t$ normalized if $t=t \downarrow$.

Without loss of generality, we will consider for our calculus only starting configurations $\langle A, R, \emptyset\rangle$ where $A, R$ are as above.

The calculus assumes the availability of a procedure for checking entailment in the (decidable) theory of linear integer arithmetic $\left(\left.\right|_{\text {LIA }}\right)$. The only significant

\footnotetext{
5 Each negative membership constraint $s \notin r$ can be replaced by $s \in r^{\mathrm{c}}$ where $r^{\mathrm{c}}$ is a regular expression generating the complement of $\mathcal{L}(r)$. This replacement is effective although current procedures for computing $r^{\mathrm{c}}$ are generally inefficient in practice.

6 The system is not confluent but we do not need it to be.
} 
deviation we require is that the procedure be able to accept terms of the form $|x|$, where $x$ is a string variable, by treating the whole term as an arithmetic variable.

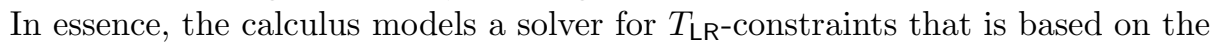
cooperation of a standard subsolver for linear arithmetic constraints and a novel subsolver that processes membership constraints natively, without reduction to automata problems. This is done by processing regular expressions by means of algebraic manipulations and non-deterministic choices. The two subsolvers communicate by exchanging linear arithmetic constraints over string lengths.

Derivation rules The rules of the calculus are provided in Figure 4 in guarded assignment form where fields $\mathrm{A}, \mathrm{R}$, and $\mathrm{V}$ store, in order, the components of a current configuration $\langle A, R, V\rangle$. A derivation rule applies to a current configuration $C$ if all of the rule's premises hold for $C$ and the resulting configuration is different from $C$. A rule's conclusion describes how each component of $C$ is changed, if at all. In the rules, we write $S, t$ as an abbreviation for $S \cup\{t\}$. Rules with two or more conclusions separated by the symbol $\|$ are non-deterministic branching rules.

The derivation rules rely on several computable functions and predicates, described below and defined formally in Figures 5, 6, 7, 8, and 9, which apply to $\sqcap$-free regular expressions.

- The family of functions $\left(\partial_{c}\right)_{c \in \mathcal{A}}$ computes the partial derivative of the input with respect to character $c$. Concretely, $\partial_{c}(r)$ is a regular expression whose language is the set of all words $w$ (including the empty one) such that $c w \in$ $\mathcal{L}(r)$.

- The predicate $\varepsilon$ holds exactly for those regular expressions whose language contains the empty string $\epsilon$.

- The function $\gamma$ produces three outputs from a normalized regular expression $r$ with top symbol other than $\varnothing$ or $\sqcup$ : a solved-form term $q$, an arithmetic term $u$, and a set $A$ of arithmetic constraints over the (integer) variables in $q$ and $u$. Intuitively, $u$ and $A$ together express constraints on the possible lengths of the words in $\mathcal{L}(r)$.

- The function $\beta$ returns a finite set of regular expression pairs. Each pair $\left(r_{1}, r_{2}\right) \in \beta(r)$ is such that $\mathcal{L}(r)=\mathcal{L}\left(r_{1} \cdot r_{2}\right)$. Moreover, $\beta(r)$ is exhaustive in the sense that for every pair of words $w_{1}, w_{2}$ such that $w_{1} w_{2} \in \mathcal{L}(r)$, there is a pair $\left(r_{1}, r_{2}\right) \in \beta(r)$ such that $w_{1} \in \mathcal{L}\left(r_{1}\right)$ and $w_{2} \in \mathcal{L}\left(r_{2}\right)$.

The definition of the partial derivative functions is due to Antimirov [2]; the functions $\gamma$ and $\beta$ are novel. Given these auxiliary predicates and functions, the calculus rules should be self-explanatory, with the possible exception of Assign-2. This rule considers a membership constraint $(x$ in $r$ ) where $r$ is not a union and (by construction) contains no occurrences of $\varnothing$ and $\sqcap$. If $x$ occurs in no other membership constraints in the $\mathrm{R}$ component of the configuration, the rule uses $\gamma$ to compute a solution form of $(x$ in $r)$ and stores it in the $\mathrm{V}$ component.

Derivation trees and derivations The rules in this calculus are used to construct derivation trees. A derivation tree is a tree where each node is a configuration and each non-root node is obtained from its parent node by applying 


$$
\begin{gathered}
\varepsilon(r) \quad \text { iff } \quad\left(r=r_{1} \cdot r_{2} \text { and } \varepsilon\left(r_{1}\right) \text { and } \varepsilon\left(r_{2}\right)\right) \text { or } r=\epsilon \text { or } r=r_{1}^{*} \text { or } \\
\left(r=r_{1} \sqcup r_{2} \text { and } \varepsilon\left(r_{1}\right)\right) \text { or }\left(r=r_{1} \sqcup r_{2} \text { and } \varepsilon\left(r_{2}\right)\right)
\end{gathered}
$$

Fig. 5. Definition of predicate $\varepsilon$.

$$
\begin{aligned}
& \partial_{c} \varnothing=\varnothing \\
& \partial_{c}\left(r_{1} \sqcup r_{2}\right)=\partial_{c} r_{1} \sqcup \partial_{c} r_{2} \\
& \partial_{c}(c \cdot s)=s \\
& \partial_{c} \epsilon=\varnothing \\
& \partial_{c}\left(r_{1} \cdot r_{2}\right)=\left(\partial_{c} r_{1} \cdot r_{2}\right) \sqcup \partial_{c} r_{2} \quad \text { if } \varepsilon\left(r_{1}\right) \\
& \partial_{c} \mathrm{Ch}=\epsilon \\
& \partial_{c}\left(r_{1} \cdot r_{2}\right)=\partial_{c} r_{1} \cdot r_{2} \quad \text { if not } \varepsilon\left(r_{1}\right) \\
& \partial_{c}\left(r^{*}\right)=\left(\partial_{c} r\right) \cdot r^{*} \\
& \partial_{c}(d \cdot s) \quad=\varnothing \quad \text { if } c \neq d
\end{aligned}
$$

Fig. 6. Definition of partial derivative function $\partial_{c}$.

one of the derivation rules. We call the root of a derivation tree an initial configuration. A branch of a derivation tree is saturated if no rules apply to its leaf, it is closed if it ends with unsat. A derivation tree is closed if all of its branches are closed.

A derivation tree derives from a derivation tree $T$ if it is obtained from $T$ by the application of exactly one of the derivation rules to one of $T$ 's leaves. A derivation is a sequence $\left(T_{i}\right)_{i \geq 0}$ of derivation trees such that $T_{0}$ is a one-node tree whose root is an initial configuration and $T_{i+1}$ derives from $T_{i}$ for all $i \geq 0$.

Let $S$ be a set of $\Sigma_{\mathrm{LR}}$-constraints. A refutation of set $S$ is a derivation that starts with a one-node tree with a configuration $\langle A, R, \emptyset\rangle$ where $A \cup R$ is $T_{\mathrm{LR}^{-}}$ equisatisfiable with $S$, and ends with a closed tree.

Example 1. Consider the satisfiable initial configuration with $\mathrm{A}=\emptyset, \mathrm{V}=\emptyset$, and $\mathrm{R}=\left\{b c \cdot x\right.$ in $\left.\left((a a \sqcup b)^{*} \cdot c\right)^{*} \sqcup a \cdot c^{*}\right\}$ where $x$ is a variable of sort String and $a, b, c$ are characters. A derivation in the calculus can start with an application of the Union rule. In the branch $b c \cdot x$ in $a \cdot c^{*}$, Consume- 2 will apply and replace the constraint with $c \cdot x$ in $\varnothing$ which then will be closed by EmptyR. In the branch $b c \cdot x$ in $\left((a a \sqcup b)^{*} \cdot c\right)^{*}$, Consume-2 will be applied twice: once for $b$, resulting in $\mathrm{R}=\left\{c \cdot x\right.$ in $\left.(a a \sqcup b)^{*} \cdot c \cdot\left((a a \sqcup b)^{*} \cdot c\right)^{*}\right\}$; and once for $c$, resulting in $\mathrm{R}=$ $\left\{x\right.$ in $\left.\left((a a \sqcup b)^{*} \cdot c\right)^{*}\right\}$. Now, by applying Assign-2 to the resulting configuration, we will have the following saturated configuration:

$$
\begin{aligned}
& \mathrm{A}=\left\{z_{1} \geq 0, z_{2} \geq 0, z_{3} \geq 0, z_{4} \geq 0, z_{1} \approx 0 \Rightarrow z_{2} \approx 0\right\} \quad \mathrm{R}=\emptyset \quad \mathrm{V}=\left\{x \text { in } q_{2}\right\} \\
& \quad \cup\left\{z_{2} \approx z_{3}+z_{4},|x| \approx 2 * z_{3}+z_{4}+z_{1}\right\}
\end{aligned}
$$

where $q_{2}=\operatorname{sh}\left(q_{1}, c^{z_{1}}, r_{1}\right), q_{1}=\operatorname{sh}\left((a a)^{z_{3}}, b^{z_{4}}, r_{2}\right), r_{1}=\left((a a \sqcup b)^{*} \cdot c\right)^{z_{1}}, r_{2}=(a a \sqcup$ $b)^{z_{2}}$, and $z_{1}, \ldots, z_{4}$ are fresh variables of sort Int. The set in $\mathrm{A}$ is satisfiable, for instance with the variable assignment $\left\{z_{1} \mapsto 1, z_{2} \mapsto 2, z_{3} \mapsto 1, z_{4} \mapsto 1,|x| \mapsto 4\right\}$. Given this assignment one can evaluate - deterministically - the term $q_{2}$ inside out and obtain $q_{2}=\{a a b c, b a a c\}$ after evaluating $q_{1}$ to $\{a a b, b a a\}$. At this point, any element of $q_{2}$ is a solution for $x$ in the original problem. As we show later, any other satisfying assignment for A will lead to a ground expression for $q_{2}$ that is guaranteed to generate a non-empty language of solutions for $x$. 


$$
\begin{array}{rlrl}
\beta(\varnothing) & =\emptyset \quad \beta(c)=\{(c, \epsilon),(\epsilon, c)\} \quad \beta\left(r_{1} \sqcup r_{2}\right)=\beta\left(r_{1}\right) \cup \beta\left(r_{2}\right) \\
\beta(\epsilon) & =\{(\epsilon, \epsilon)\} \quad \beta(\mathrm{Ch})=\{(\mathrm{Ch}, \epsilon),(\epsilon, \mathrm{Ch})\} & \\
\beta\left(r^{*}\right) & =\beta(\epsilon) \cup\left\{\left(r^{*} \cdot r_{1}, r_{2} \cdot r^{*}\right) \mid\left(r_{1}, r_{2}\right) \in \beta(r)\right\} \\
\beta\left(r_{1} \cdot r_{2}\right) & =\left\{\left(r_{11}, r_{12} \cdot r_{2}\right) \mid\left(r_{11}, r_{12}\right) \in \beta\left(r_{1}\right)\right\} \cup\left\{\left(r_{1} \cdot r_{21}, r_{22}\right) \mid\left(r_{21}, r_{22}\right) \in \beta\left(r_{2}\right)\right\}
\end{array}
$$

Fig. 7. Definition of splitting function $\beta$.

$$
\begin{aligned}
& \pi\left(r, r^{\prime}\right)=\pi^{\prime}\left(r, r^{\prime}, \emptyset\right) \quad \pi^{\prime}\left(r, r^{\prime}, C\right)=y_{r, r^{\prime}} \text { if } y_{r, r^{\prime}} \in C \quad \pi^{\prime}(r, \varnothing, C)=\varnothing \\
& \pi^{\prime}(\epsilon, r, C)=\epsilon \text { if } \varepsilon(r) \quad \pi^{\prime}(\epsilon, r, C)=\varnothing \text { if not } \varepsilon(r) \quad \pi^{\prime}(\varnothing, r, C)=\varnothing \\
& \pi^{\prime}(r, \epsilon, C)=\epsilon \text { if } \varepsilon(r) \quad \pi^{\prime}(r, \epsilon, C)=\varnothing \text { if not } \varepsilon(r) \quad \pi^{\prime}(r, r, C)=r \\
& \pi^{\prime}\left(r, r^{\prime}, C\right)=r_{1}^{*} \cdot r_{1}^{\prime} \text { if } v_{r, r^{\prime}} \notin C \text { and } \varepsilon(r) \text { and } \varepsilon\left(r^{\prime}\right) \text { where } \\
& \left(r_{1}, r_{1}^{\prime}\right)=\rho_{v_{r, r^{\prime}}}\left(\epsilon \sqcup \bigsqcup_{c \in \mathcal{A}} c \cdot \pi^{\prime}\left(\partial_{c} r, \partial_{c} r^{\prime}, C^{\prime}\right)\right), \quad C^{\prime}=C \cup\left\{v_{r, r^{\prime}}\right\} \\
& \pi\left(r, r^{\prime}, C\right)=r_{1}^{*} \cdot r_{1}^{\prime} \text { if } v_{r, r^{\prime}} \notin C \text { and not }\left(\varepsilon(r) \text { and } \varepsilon\left(r^{\prime}\right)\right) \text { where } \\
& \left(r_{1}, r_{1}^{\prime}\right)=\rho_{v_{r, r^{\prime}}}\left(\bigsqcup_{c \in \mathcal{A}} c \cdot \pi^{\prime}\left(\partial_{c} r, \partial_{c} r^{\prime}, C^{\prime}\right)\right), \quad C^{\prime}=C \cup\left\{v_{r, r^{\prime}}\right\} \\
& \rho_{y}(\varnothing)=(\varnothing, \varnothing) \quad \rho_{y}(y)=(\epsilon, \varnothing) \quad \rho_{y}(r)=(\epsilon, r) \text { if } y \notin \mathcal{V}(r) \\
& \rho_{y}(r)=\left(r_{1} \cdot r_{21}, r_{22}\right) \text { if } y \in \mathcal{V}(r), r=r_{1} \cdot r_{2} \text {, and }\left(r_{21}, r_{22}\right)=\rho_{y}\left(r_{2}\right) \\
& \rho_{y}(r)=\left(r_{11} \sqcup r_{21}, r_{12} \sqcup r_{22}\right) \text { if } y \in \mathcal{V}(r), r=r_{1} \sqcup r_{2} \text {, and }\left(r_{i 1}, r_{i 2}\right)=\rho_{y}\left(r_{i}\right)
\end{aligned}
$$

Fig. 8. Definition of intersection function $\pi$.

Example 2. Suppose we start with the unsatisfiable configuration with $\mathrm{A}=$ $\{|x| \approx 2 * k+1\}, \mathrm{R}=\{x \cdot x$ in $r\}$, and $\mathrm{V}=\emptyset$ where $r=(a a a a)^{*}$ and $a$ is a character. One possibility is to apply the Split rule. Since $\beta(r)=\{(\epsilon, \epsilon),(r$. $a, a a a \cdot r),(r \cdot a, a a a \cdot r),(r \cdot a a, a a \cdot r),(r \cdot a a a, a \cdot r)\}$, four branches will be created. In the first branch, $\mathrm{R}=\{x$ in $\epsilon\}$. The rule Assign-1 can be applied, adding $x$ in $\epsilon$ to $\mathrm{V}$ and $|x| \approx 0$ to A. After that, the branch can be closed by A-Conflict. In the second branch, $\mathrm{R}=\{x$ in $r \cdot a, x$ in $a a a \cdot r\}$ to which Inter can be applied, replacing the constraints in $\mathrm{R}$ with $x$ in $\varnothing$. Then the branch can be closed by EmptyS. Something, similar can be done on the fourth branch. In the third branch, $\mathrm{R}$ can become $\{x$ in $a a \cdot r\}$ by Inter. Then $|x| \approx 2+4 * z$ and $z \geq 0$ can be added to A by Assign-2, with $z$ a fresh integer variable. That branch can be closed by A-Conflict, yielding a refutation of the input problem.

\section{Calculus Correctness}

We prove the correctness of the calculus in by showing that $(i)$ it has no infinite derivations; (ii) its rules preserve satisfiability in $T_{\mathrm{LR}}$; (iii) every saturated branch in a derivation tree determines a model of $T_{\mathrm{LR}}$ that satisfies the initial configuration. Together with the termination of the auxiliary functions and pro- 


$$
\begin{aligned}
\gamma(r)= & \gamma^{\prime}(r, \emptyset) \\
\gamma^{\prime}(l, A)= & (l,|l| \downarrow, A) \quad \gamma^{\prime}(\mathrm{Ch}, A)=(\mathrm{Ch}, 1, A) \\
\gamma^{\prime}\left(r_{1} \cdot r_{2}, A\right)= & \left(q_{1} \cdot q_{2}, u_{1}+u_{2}, A_{1} \cup A_{2}\right) \text { where }\left(q_{i}, u_{i}, A_{i}\right)=\gamma^{\prime}\left(r_{i}, A\right) \text { for } i=1,2 \\
\gamma^{\prime}\left(r^{*}, A\right)= & \left(q, u, B \cup\left\{z_{1} \geq 0\right\}\right) \text { where }(q, u, B)=\gamma^{\prime}\left(r^{z_{1}}, A\right) \\
\gamma^{\prime}\left(l^{z}, A\right)= & \left(l^{z}, z \times|l| \downarrow, A\right) \quad \gamma^{\prime}\left(\mathrm{Ch}^{z}, A\right)=\left(\mathrm{Ch}^{z}, z, A\right) \\
\gamma^{\prime}\left(\left(r_{1} \sqcup r_{2}\right)^{z}, A\right)= & \left(\operatorname{sh}\left(q_{1}, q_{2},\left(r_{1} \sqcup r_{2}\right)^{z}\right), u_{1}+u_{2}, B\right) \\
& \quad \text { where } B=A_{1} \cup A_{2} \cup\left\{z \approx z_{1}+z_{2}, z_{1} \geq 0, z_{2} \geq 0\right\} \\
\gamma^{\prime}\left(\left(r_{1} \cdot r_{2}\right)^{z}, A\right)= & \left(\operatorname{sh}\left(q_{1}, q_{2},\left(r_{1} \cdot r_{2}\right)^{z}\right), \gamma_{1}+u_{2}, A_{1} \cup A_{2}\right) \\
& \text { where }\left(q_{i}, u_{i}, A_{i}\right)=\gamma^{\prime}\left(r_{i}^{z}, A\right) \text { for } i=1,2 \\
\gamma^{\prime}\left(\left(r^{*}\right)^{z}, A\right)= & \gamma^{\prime}\left(q, u, B \cup\left\{z \approx 0 \Rightarrow z_{1} \approx 0, z_{1} \geq 0\right\}\right) \text { where }(q, u, B)=\gamma^{\prime}\left(r^{z_{1}}, A\right)
\end{aligned}
$$

Fig. 9. Definition of function $\gamma$. The letters $z_{1}$ and $z_{2}$ denote fresh integer variables variables variables variables variables.

cedures used by the calculus, this implies the decidability of the quantifier-free satisfiability problem for $T_{\mathrm{LR}} 7^{7}$

\subsection{Termination}

Proving the termination of the auxiliary functions and predicates is a simple exercise.

Proposition 1. The function $\pi$ is well defined and computable over the set of all regular expressions. The predicate $\varepsilon$ and the functions $\partial_{c}, \beta$ and $\gamma$ are well defined and computable over the set of all $\sqcap$-free regular expressions.

By Proposition 1, every rule is effective. To prove the termination of the calculus it suffices to define a well-founded ordering of configurations and show that every rule application produces a smaller configuration along that ordering.

Proposition 2. Every derivation in the calculus is finite.

Proof (Sketch). One can show that every application of a derivation rule to a leaf of a derivation tree produces smaller configurations with respect to a wellfounded relation $\succ$ over configurations which implies that no derivation tree can be grown indefinitely.

The relation $\succ$ is defined as follows. To each configuration $\langle A, R, V\rangle$ we associate a tuple $(\mathcal{V}(R), \operatorname{ms}(R), \operatorname{occ}(R))$ where $\operatorname{ms}(R)$ is the multiset $\{s \mid s$ in $r \in \mathrm{R}\}$ and $\operatorname{occ}(R)$ is the number of occurrences of $\sqcup$ in R. Let $\succ$ str be the ordering over

\footnotetext{
${ }^{7}$ For space constraints, the most of the proofs of these results are omitted. The interested reader is referred to the longer version of this paper 20] for the missing proofs.
} 
string terms such that $s \succ_{\mathrm{Str}} t$ iff $s$ has a greater term size than $t$, with the convention that $\epsilon$ has size 0 . Let $\succ_{\text {lex }}$ be the lexicographic extension of the following orderings to tuples like $(\mathcal{V}(R), \operatorname{ms}(R)$, occ $(R))$ above: the set inclusion ordering; the multiset ordering extending $\succ_{\text {Str }}$; the $>$ ordering over natural numbers. Finally, define $\succ$ where $(i)\left\langle A_{1}, R_{1}, V_{1}\right\rangle \succ\left\langle A_{2}, R_{2}, V_{2}\right\rangle$ iff $\left(\mathcal{V}\left(R_{1}\right), \operatorname{ms}\left(R_{1}\right), \operatorname{occ}\left(R_{1}\right)\right) \succ_{\text {lex }}$ $\left(\mathcal{V}\left(R_{2}\right), \operatorname{ms}\left(R_{2}\right), \operatorname{occ}\left(R_{2}\right)\right)$ and $(i i)\langle A, R, V\rangle \succ$ unsat. The well foundedness of $\succ$ follows by standard results (see e.g., [3]).

\subsection{Correctness}

To prove the correctness of the calculus we use the following properties of the various auxiliary functions.

Lemma 1 (Correctness of Normalization). Every rule in Figure 3 preserves term equivalence in $T_{\mathrm{LR}}$.

Lemma 2 (Correctness of $\pi$ ). For any regular expressions $r_{1}$ and $r_{2}, \pi\left(r_{1}, r_{2}\right)$ contains no occurrences of $\sqcap$. Moreover, $\mathcal{L}\left(\pi\left(r_{1}, r_{2}\right)\right)=\mathcal{L}\left(r_{1} \sqcap r_{2}\right)$.

Lemma 3. For all normalized regular expressions $r$ and for all characters $c \in$ $\mathcal{A}$, the following hold:

1. $\varepsilon(r)$ iff $\epsilon \in \mathcal{L}(r)$;

2. $\mathcal{L}\left(\partial_{c} r\right)=\{w \mid c w \in \mathcal{L}(r)\}$;

3. for all $\left(r_{1}, r_{2}\right) \in \beta(r), \mathcal{L}\left(r_{1} \cdot r_{2}\right)=\mathcal{L}(r)$;

4. for all $w_{1} w_{2} \in \mathcal{L}(r)$, there is $a\left(r_{1}, r_{2}\right) \in \beta(r)$ s.t. $w_{1} \in \mathcal{L}\left(r_{1}\right)$ and $w_{2} \in \mathcal{L}\left(r_{2}\right)$.

Lemma 4. Let $x$ be a string variable, let $r$ be a normalized regular expression with $\operatorname{top}(r) \notin\{\varnothing, \sqcup\}$, let $A$ be a set of arithmetic constraints, and let $\left(r_{\gamma}, u_{\gamma}, A_{\gamma}\right)=\gamma(r)$.

1. The constraint set $S:=\{x$ in $r\} \cup A$ is satisfied by a model $\mathcal{I}$ of $T_{\mathrm{LR}}$ iff the set $S_{\gamma}:=\left\{x\right.$ in $\left.r_{\gamma},|x| \approx u_{\gamma}\right\} \cup A \cup A_{\gamma}$ is satisfied by a model $\mathcal{I}_{\gamma}$ of $T_{\mathrm{LR}}$ where $\mathcal{I}$ and $\mathcal{I}_{\gamma}$ agree on the variables of $S$.

2. All models $\mathcal{I}$ of $T_{\mathrm{LR}}$ satisfying $A_{\gamma}$ are such that for all $w \in r_{\gamma}^{\mathcal{I}}$, the length of $w$ equals $u_{\gamma}^{\mathcal{I}}$.

We say that a configuration $\langle A, R, V\rangle$ is satisfied by an interpretation $\mathcal{I}$ if the set $A \cup R \cup V$ is satisfied by $\mathcal{I}$. We consider unsat to be satisfied by no interpretation.

Lemma 5. For every rule of the calculus, the premise configuration is satisfied by a model $\mathcal{I}_{p}$ of $T_{\mathrm{LR}}$ iff one of its conclusion configurations is satisfied by a model $\mathcal{I}_{c}$ of $T_{\mathrm{LR}}$ where $\mathcal{I}_{p}$ and $\mathcal{I}_{c}$ agree on the variables shared by the two configurations.

Using the previous lemma in the left-to-right direction together with a structural induction argument on derivation trees, one can readily show that the root of every closed derivation tree is unsatisfiable. From this, the refutation soundness of the calculus easily follows. 
Proposition 3 (Refutation Soundness). Every set of $T_{\mathrm{LR}}$-constraints that has a refutation is $T_{\mathrm{LR}}$-unsatisfiable.

Thanks to earlier lemmas and the one below one can also prove that the calculus is solution sound.

Lemma 6. If $\langle A, R, V\rangle$ is a saturated leaf of a derivation tree with root $\left\langle A_{0}, R_{0}, \emptyset\right\rangle$ then for every (string) variable $x$ in $R_{0}$ there is a constraint of the form ( $x$ in $q$ ) in $V$.

Proposition 4 (Solution Soundness). For every saturated leaf $\langle A, R, V\rangle$ of a derivation tree with root $\left\langle A_{0}, R_{0}, \emptyset\right\rangle$ there is a model $\mathcal{I}$ of $T_{\mathrm{LR}}$ that satisfies $A_{0} \cup R_{0}$ and is such that $x^{\mathcal{I}} \in q^{\mathcal{I}}$ for all $(x$ in $q) \in V$.

Proof. Let $K:=\langle A, R, V\rangle$ be as above. It is not difficult to show based the derivation rules that $\mathcal{V}\left(A_{0} \cup R_{0}\right) \subseteq \mathcal{V}(A \cup R \cup V)$ and $A_{0} \subseteq A$. Moreover, every integer variable of $V$ is in $A$, by definition of $\gamma$, and each string variable of $R$ occurs in $V$ exactly once.

The set $R$ contains at most constraints of the form $(\epsilon$ in $r)$ with $\epsilon \in \mathcal{L}(r)$; otherwise, one of the derivation rules would apply to $K$, against the assumption that it is saturated. This makes $R$ trivially satisfiable. The set $A$ is satisfiable as well, otherwise A-Conflict would apply. Let $\mathcal{J}$ be a model of $T_{\mathrm{LR}}$ satisfying $A$ and let $(x$ in $q)$ be any element of $V$. We claim that the set $q^{\mathcal{J}}$ is nonempty and contains only words of length $|x|^{\mathcal{J}}$. In fact, if $(x$ in $q)$ was added to $V$ by Assign-1, then $q$ is a literal $l$ and $|x| \approx|l| \downarrow \in A$. If $(x$ in $q$ ) was added to $V$ by Assign-2, then $\gamma(r)=\left(q, u_{\gamma}, A_{\gamma}\right)$ for some $r$, where $A_{\gamma} \subseteq A$ and $|x| \approx u_{\gamma} \in A$. Since $\mathcal{J}$ satisfies $A_{\gamma}$, by Lemma 4 2 , all words in $q^{\mathcal{J}}$, if any, are of length $u_{\gamma}^{\mathcal{J}}$ which is the same as $|x|^{\mathcal{J}}$. To argue that $q^{\mathcal{J}}$ is non-empty, by Lemma 4, 2), it is enough to argue that $\mathcal{L}(r)$ is nonempty. This can be seen by observing that, by definition of the the rewrite rules in Figure 3, and by Lemma 1 and Lemma 2, $r$ is guaranteed to contain no occurrences of $\varnothing$ or $\sqcap$, and containing such symbols is a necessary condition for a regular expression to have an empty language. The statement of the lemma follows by the generality of $(x$ in $q)$.

Proposition 5 (Refutation Completeness). Every set of $T_{\mathrm{LR}}$-constraints unsatisfiable in $T_{\mathrm{LR}}$ has a refutation.

Proof. Contrapositively, suppose that the set of $T_{\mathrm{LR}}$-constraints does not have a refutation. Then, by Proposition 2 , it must have a derivation that generates a tree with a saturated branch. By Proposition 4 the set is satisfiable in $T_{\mathrm{LR}}$.

\subsection{Decidability}

Proposition 6 (Decidability). The $T_{\mathrm{LR}}$-satisfiability of quantifier-free $\Sigma_{\mathrm{LR}}$ formulas with no regular expression variables is decidable. 
Proof. By standard methods, the $T_{\mathrm{LR}}$-satisfiability of quantifier-free $\Sigma_{\mathrm{LR}}$-formulas with no variables of sort Lan can be effectively reduced to the $T_{\mathrm{LR}}$-satisfiability of $T_{\mathrm{LR}}$-constraints. The existence of a terminating procedure to check such constraints is a consequence of Proposition 1 and Proposition 2. The correctness of the procedure is a consequence of Propositions 3 and 5 .

\section{Conclusion and Further Work}

We have presented an algebraic approach for solving regular membership constraints and linear length constraints in the theory of strings. This approach works directly on regular expressions without the need to translate them to automata. Moreover, it does not require imposing any a priori length bounds on string variables. We have proved that our approach is sound, complete and terminating, thus it is a decision procedure for this fragment. In addition, when the constraints are satisfiable, our approach provides a model - in fact a generator of a set of models. Therefore, it has all the properties required for integration into an SMT solver.

In ongoing work, we are investigating a possible extension of our procedure to word equations over unbounded strings. Although the satisfiability of sets of word equations is also decidable, the decidability of the combined language is still an open problem. We hope to find a fragment that is sufficiently expressive for real-world problems, while also being decidable, or at least effective for solving problems in practice.

Additionally, we have identified two bottlenecks in the calculus presented here: the computation of the intersection and the complement operations over regular expressions. Therefore, we plan to focus on developing approaches for computing these operations that are efficient in practice. We are also working on an extension to symbolic regular expressions, specifically, regular expressions that contain string variables.

\section{References}

[1] P. A. Abdulla, M. F. Atig, Y.-F. Chen, L. Holik, A. Rezine, P. Rummer, and J. Stenman. String constraints for verification. In A. Biere and R. Bloem, editors, Proceedings of the 26th International Conference on Computer Aided Verification, volume 8559 of Lecture Notes in Computer Science. Springer, 2014.

[2] V. Antimirov. Partial derivatives of regular expressions and finite automaton constructions. Theor. Comput. Sci., 155(2):291-319, Mar. 1996.

[3] F. Baader and T. Nipkow. Term Rewriting and All That. Cambridge University Press, 1998.

[4] B. Badban and M. T. Dashti. Semi-linear parikh images of regular expressions via reduction. In Proceedings of the 35th International Conference on Mathematical Foundations of Computer Science, MFCS'10, pages 653-664, Berlin, Heidelberg, 2010. Springer-Verlag.

[5] C. Barrett, R. Sebastiani, S. Seshia, and C. Tinelli. Satisfiability modulo theories. In A. Biere, M. J. H. Heule, H. van Maaren, and T. Walsh, editors, Handbook of Satisfiability, volume 185, chapter 26, pages 825-885. IOS Press, February 2009. 
[6] G. Berry and R. Sethi. From regular expressions to deterministic automata. Theor. Comput. Sci., 48(1):117-126, Dec. 1986.

[7] N. Bjørner, N. Tillmann, and A. Voronkov. Path feasibility analysis for stringmanipulating programs. In Proceedings of the 15th International Conference on Tools and Algorithms for the Construction and Analysis of Systems: Held as Part of the Joint European Conferences on Theory and Practice of Software, ETAPS 2009, pages 307-321. Springer-Verlag, 2009.

[8] A. S. Christensen, A. Møller, and M. I. Schwartzbach. Precise analysis of string expressions. In Proceedings of the 10th International Conference on Static Analysis, SAS'03, pages 1-18, Berlin, Heidelberg, 2003. Springer-Verlag.

[9] X. Fu and C. chih Li. A string constraint solver for detecting web application vulnerability. In Proceedings of the 22nd International Conference on Software Engineering and Knowledge Engineering, SEKE'2010. Knowledge Systems Institute Graduate School, 2010.

[10] I. Ghosh, N. Shafiei, G. Li, and W.-F. Chiang. JST: An automatic test generation tool for industrial Java applications with strings. In Proceedings of the 2013 International Conference on Software Engineering, ICSE '13, pages 992-1001, Piscataway, NJ, USA, 2013. IEEE Press.

[11] J. G. Henriksen, J. L. Jensen, M. E. Jørgensen, N. Klarlund, R. Paige, T. Rauhe, and A. Sandholm. Mona: Monadic second-order logic in practice. In Proceedings of the First International Workshop on Tools and Algorithms for Construction and Analysis of Systems, TACAS '95, pages 89-110, London, UK, UK, 1995. Springer-Verlag.

[12] P. Hooimeijer and M. Veanes. An evaluation of automata algorithms for string analysis. In Proceedings of the 12th international conference on Verification, model checking, and abstract interpretation, pages 248-262. Springer-Verlag, 2011.

[13] P. Hooimeijer and W. Weimer. A decision procedure for subset constraints over regular languages. In Proceedings of the 2009 ACM SIGPLAN conference on Programming language design and implementation, pages 188-198. ACM, 2009.

[14] P. Hooimeijer and W. Weimer. Solving string constraints lazily. In Proceedings of the IEEE/ACM international conference on Automated software engineering, pages 377-386. ACM, 2010.

[15] A. Kiezun, V. Ganesh, P. J. Guo, P. Hooimeijer, and M. D. Ernst. HAMPI: a solver for string constraints. In Proceedings of the eighteenth international symposium on Software testing and analysis, pages 105-116. ACM, 2009.

[16] N. Klarlund, A. Møller, and M. I. Schwartzbach. Mona implementation secrets. In Revised Papers from the 5th International Conference on Implementation and Application of Automata, CIAA '00, pages 182-194, London, UK, UK, 2001. Springer-Verlag.

[17] D. Kozen. Lower bounds for natural proof systems. In FOCS, pages 254-266. IEEE Computer Society, 1977.

[18] G. Li and I. Ghosh. Pass: String solving with parameterized array and interval automaton. In V. Bertacco and A. Legay, editors, Hardware and Software: Verification and Testing, volume 8244 of Lecture Notes in Computer Science, pages 15-31. Springer International Publishing, 2013.

[19] T. Liang, A. Reynolds, C. Tinelli, C. Barrett, and M. Deters. A DPLL(T) theory solver for a theory of strings and regular expressions. In A. Biere and R. Bloem, editors, Proceedings of the 26th International Conference on Computer Aided Verification, volume 8559 of Lecture Notes in Computer Science. Springer, 2014. 
[20] T. Liang, N. Tsiskaridze, A. Reynolds, C. Tinelli, and C. Barrett. A decision procedure for regular membership and length constraints over unbounded strings. Technical report, Department of Computer Science, The University of Iowa, 2015. (Available at http://www.cs.uiowa.edu/ tinelli/papers.html).

[21] K. Z. M. Lu. XHaskell - Adding Regular Expression Type to Haskell. PhD thesis, National University of Singapore, 2009.

[22] G. S. Makanin. The problem of solvability of equations in a free semigroup. English transl. in Math USSR Sbornik, 32:147-236, 1977.

[23] Y. Matiyasevich. Hilbert's tenth problem and paradigms of computation. In Proceedings of the First International Conference on Computability in Europe: New Computational Paradigms, CiE'05, pages 310-321. Springer-Verlag, Berlin, Heidelberg, 2005.

[24] R. J. Parikh. On context-free languages. J. ACM, 13(4):570-581, Oct. 1966.

[25] W. Plandowski. Satisfiability of word equations with constants is in pspace. J. ACM, 51(3):483-496, May 2004.

[26] G. Rosu and M. Viswanathan. Testing extended regular language membership incrementally by rewriting. In R. Nieuwenhuis, editor, Rewriting Techniques and Applications, volume 2706 of Lecture Notes in Computer Science, pages 499-514. Springer Berlin Heidelberg, 2003.

[27] K. Schulz, editor. Word Equations and Related Topics. Springer-Verlag New York, Inc., New York, NY, USA, 1990.

[28] T. Tateishi, M. Pistoia, and O. Tripp. Path- and index-sensitive string analysis based on monadic second-order logic. ACM Trans. Softw. Eng. Methodol., 22(4):33:1-33:33, Oct. 2013.

[29] N. Tillmann and J. Halleux. Pex - white box test generation for .net. In B. Beckert and R. Hähnle, editors, Tests and Proofs, volume 4966 of Lecture Notes in Computer Science, pages 134-153. Springer Berlin Heidelberg, 2008.

[30] M.-T. Trinh, D.-H. Chu, and J. Jaffar. S3: A symbolic string solver for vulnerability detection in web applications. In M. Yung and N. Li, editors, Proceedings of the 21st ACM Conference on Computer and Communications Security, 2014.

[31] M. Veanes. Applications of symbolic finite automata. In Proceedings of the 18th International Conference on Implementation and Application of Automata, CIAA'13, pages 16-23, Berlin, Heidelberg, 2013. Springer-Verlag.

[32] M. Veanes, N. Bjørner, and L. De Moura. Symbolic automata constraint solving. In Proceedings of the 17th International Conference on Logic for Programming, Artificial Intelligence, and Reasoning, LPAR'10, pages 640-654, Berlin, Heidelberg, 2010. Springer-Verlag.

[33] F. Yu, M. Alkhalaf, and T. Bultan. Stranger: An automata-based string analysis tool for php. In J. Esparza and R. Majumdar, editors, Tools and Algorithms for the Construction and Analysis of Systems, volume 6015 of Lecture Notes in Computer Science, pages 154-157. Springer Berlin Heidelberg, 2010.

[34] Y. Zheng, X. Zhang, and V. Ganesh. Z3-str: A z3-based string solver for web application analysis. In Proceedings of the 2013 9th Joint Meeting on Foundations of Software Engineering, ESEC/FSE 2013, pages 114-124, New York, NY, USA, 2013. ACM. 